This is the peer reviewed version of the following article: Micallef, Ricarda, Grewal, Jaspal Singh, Khan, Sharifah, Wells, Joshua and Kayyali, Reem (2019) Health Champions in South London : evaluation of training, and impact on public health. International Journal of Pharmacy Practice, 27(1), pp. 71-79., which has been published in final form at https://doi.org/10.1111/ijpp.12464. This article may be used for non-commercial purposes in accordance with Wiley Terms and Conditions for Self-Archiving. 


\section{Health Champions in South London: evaluation of training, and impact on}

public health

Miss Ricarda Micallef, MRPharmS, PhD candidate. ${ }^{a}$

Jaspal Singh Grewal, MSc Student. ${ }^{a}$

Sharifah Khan, MSc Student. ${ }^{a}$

Joshua Wells, MPharm Student. ${ }^{\text {a }}$

Reem Kayyali, MRPharmS, MSc, PhD. ${ }^{a}$

a. Kingston University, Department of Pharmacy, Penrhyn Road, Kingston Upon Thames, Surrey, KT1 2EE

Correspondence: Professor Reem Kayyali, Kingston University, Department of Pharmacy, Penrhyn Road, Kingston Upon Thames, Surrey, KT1 2EE

\section{r.kayyali@kingston.ac.uk}

\section{Contributions:}

Ricarda Micallef contributed to data analysis and drafting of manuscript.

Jaspal Singh Grewal contributed to study design and interview data collection.

Sharifah Khan contributed to study design and questionnaire data collection.

Joshua Wells contributed to drafting of manuscript.

Reem Kayyali contributed to study design, data analysis, drafting and proof reading of manuscript.

\section{Declarations:}

\section{Conflict of interest}

The Authors declare that they have no conflicts of interest to disclose.

\section{Funding}

"This research received no specific grant from any funding agency in the public, commercial, or not-for-profit sectors"

\section{Acknowledgements}

The authors would like to thank Health Education South London for funding the training events and to all of those who participated in interviews and the Health Champions for completion of the surveys. 


\section{Abstract}

Objectives: The Healthy Living Pharmacy framework, introduced in England in 2008, provides a tailored approach to the implementation of pharmacy services locally, facilitated by qualified 'Health Champions' (HCs). The study aimed to evaluate the perceived value of the one-day level $2 \mathrm{HC}$ training by assessing knowledge and confidence of $\mathrm{HC}$ pre and post-training, and changes in practice. The views of additional stakeholders on factors that either obstructed or facilitated pharmacy engagement are also explored.

Methods: This study used a mixed method approach. Pre and post-training surveys evaluating $\mathrm{HC}$ pre-existing knowledge and understanding of their role were used. Additionally, qualitative semi-structured interviews were undertaken with four key groups: participating pharmacists, non-participating pharmacists, Local Pharmaceutical Committee leads and Public Health Leads from Public Health England.

Key Findings: A total of 354 pre-training evaluation surveys were completed on the training day (100\%), compared to 54 post-evaluation postal surveys with a $19 \%$ $(n=54 / 282)$ response rate. The post-evaluation of the training indicated that $83 \%$ ( $n=45)$ of qualified HC were actively implementing their new role, with observed increased confidence and service provision. From the 22 interviews conducted two major themes emerged including: Training and Impact. Training had a positive impact on staff knowledge and confidence plus service delivery.

Conclusion: An immediate impact was observed in increasing $\mathrm{HC}$ knowledge and confidence, service delivery and awareness of facilities for patient sign-posting. There was no statistical evidence to support a positive effect stemming from training 
on services. However, it was identified that time and further training were needed to both provide and assess value within local public health. 


\section{Introduction}

With the ever increasing financial and time pressures on the National Health Service (NHS) mirrored by rising health inequalities across the UK, the value of optimising service delivery has become a key objective for all healthcare providers nationally including community pharmacy.

Community pharmacy was identified as a key provider of public health in 1986 by the Nuffield committee report. ${ }^{1}$ As far back as 1998,21 pharmacy related public health priorities e.g. smoking, alcohol and drugs were identified in the government white paper 'Our healthier nation'. ${ }^{2}$ In 2003 , the Department of Health (DoH) released a document 'A Vision for Pharmacy in the new NHS', which recognised the role pharmacists play in patient care, including public health. ${ }^{3}$ In 2005 , the community pharmacy contractual framework was established outlining three levels of services. The enhanced (locally commissioned) services, in England, are delivered based on local population needs highlighting the perceived value of targeted commissioning in reducing health inequalities. ${ }^{4}$ These services have been driven by Public Health England (PHE) and Borough councils, with the setup of Health and Wellbeing Boards (HWBs) with the aid of the pharmaceutical needs assessments (PNAs) to support effective commissioning of services. ${ }^{5,6}$ Since then, the value of community pharmacy in public health services provision has been further recognised as outlined in the NHS England's Five Year Forward View. ${ }^{7}$ Recent systemic reviews concluded that community pharmacies should be commissioned to help deliver public health services and need to work towards enhancing their contribution to public health services in that regard..$^{8,9}$ This is crucial in order to reduce the life years lost in early death and disability by the UK's major killers; cancer, coronary heart disease (CHD) and diabetes, and treat individuals holistically. ${ }^{10,11}$ 
Following the 2008 white paper, 'Pharmacy in England: Building on strengths, delivering the future,' recognising community pharmacy contribution to public health, ${ }^{12}$ NHS Portsmouth and the Local Pharmaceutical Committee (LPC) of Hampshire \& Isle of Wight developed the concept of Healthy Living Pharmacies (HLP). ${ }^{13}$ The HLP concept is a tiered framework whereby community pharmacies in England deliver health and wellbeing services based on local needs with three levels of activity which are promotion, prevention and protection and a set of three enablers; workforce development, appropriately equipped premises and local stakeholders' engagement.

One of the key components for HLP accreditation includes a trained staff member as a Health Champion (HC). The $\mathrm{HC}$ helps customers to adopt healthier lifestyles in areas such as smoking, alcohol, blood pressure, weight management and sexual health plus signposting to other community services e.g. charities, when required. The data suggested the framework could be successfully implemented with the hope of mirroring the increased and improved delivery of services. ${ }^{14}$ The successful pilot in Portsmouth created a platform for the DoH to develop a national framework and a pathfinder programme was implemented. During the pilot, the role of HCs in service delivery for HLPs was demonstrated when comparing against non-HLPs with improved performance in areas such as smoking cessation, alcohol awareness and weight management. ${ }^{15}$ The results indicated that support staff can be used effectively in public health delivery from community pharmacy. ${ }^{16}$

Following the support of the pathfinder programme in HLP implementation, Health Education South London (HESL) funded HC training across 10 boroughs throughout 
South London with 583 pharmacies selected. South London has 12 boroughs; Greenwich, Lambeth, Lewisham, Richmond, Southwark and Wandsworth, Bexley, Bromley, Croydon, Kingston, Merton and Sutton, covered by 5 LPC areas. Lambeth and Lewisham were excluded from this training as the pilot for the training had taken place there resulting in 163 accredited HCs: 71 in Lewisham and 92 in Lambeth. The project across 10 boroughs entailed training two members of staff per pharmacy as accredited HCs, although the pharmacies themselves were not HLP accredited. The training provided earned the staff member a Level 2 Understanding Health Improvement Award from the Royal Society of Public Health. Local PHE and LPC representatives attended the training days.

The one-day face-to-face training covered key areas including: UK health inequalities, effective communication in promotion of health and wellbeing, as well as highlighting the relationship between healthy lifestyle and behavioural changes. The expectation for accredited $\mathrm{HC}$ in their workplace was to provide customers with more information, to practically increase public health by utilising signpost organisations, liaising with other HCs and the continual promotion of health campaigns.

The aim of the study takes a two-dimensional approach to evaluating $\mathrm{HC}$ training in South London between April and July 2014. The study aimed to evaluate the perceived value of the one-day level $2 \mathrm{HC}$ training by assessing knowledge and confidence of $\mathrm{HC}$ before training with a comparison post-training, and changes in practice. In addition to HCs, the views of the following stakeholders; PHE leads, LPC leads and pharmacists, on factors that either obstructed or facilitated pharmacy engagement are explored. 


\section{Methods}

\section{Design:}

A mixed method approach was used; using pre and post-training questionnaires for $\mathrm{HC}$ trainees along with semi-structured interviews for stakeholders. The questionnaires were validated as part of the Lambeth/Lewisham pilot study. ${ }^{17}$ The pre-training evaluation, consisted of demographic information and nine further questions arranged in four parts as follows: Part 1: Current provision of public health services, 5 questions with tick box responses, part 2: perceived role of an $\mathrm{HC}, 1$ question with a free text box, part 3: confidence in, and value of the role, 3 questions with 5 point Likert style, part 4: public health priorities, 1 question with a free text box and part 5: demographics.

The post-training evaluation consisted of demographic information to confirm pairing and 12 questions to investigate the impact of $\mathrm{HC}$ training on the daily working activity of each participant. Where possible questions were used from the pre-training questionnaire to allow comparison. The structure of the questionnaire was as follows: Part 1: change in public health interventions, 4 questions with tick box and free text boxes, part 2: level of support, 1 question using a free text box, part 3:confidence in providing services and the role, with 4 questions using 5 point Likert scale and one open text box and part 4: future services for the pharmacy, 3 questions using 2 dichotomous questions and one open text box.

A semi-structured interview schedule was designed, with a standardised introduction format. There were then defined questions for the target groups, which can be seen 
in box 3 . The interview schedule received face validity from two pharmacists working outside of the study area.

\section{Data Collection:}

Participants from 299 pharmacies (354 in total) who attended the HC training were required to complete the pre-evaluation at the training day, with the purpose explained by the facilitators, and completed forms returned to the researcher. Posttraining surveys were posted at their work address to the $282(80 \%)$ of $\mathrm{HC}$, who had had a minimum of $21-30$ days to implement their new role along with a participant information sheet. The recommended sample size required to allow for the results to be standardisable at $95 \%$ confidence level and $5 \%$ margin of error was calculated using the Raosoft calculator, as 186 for the pre-evaluation survey and 163 for the post training survey. . During the initial training process, participants given a unique study id to allow paired comparison of pre and post-training questionnaire responses. Those who did not reply within two weeks were phoned at their place of employment to determine their reasons for not completing the post-training evaluation questionnaire. Should the participant show interest, a personal email address was documented, and another questionnaire was distributed via email. Implied consent was given through completion of the questionnaires.

For the interviews, the following key stakeholders were included: PHE leads, LPC leads and pharmacists from participating and non-participating pharmacies. All were invited via an invitation email containing information about the project. All PHE leads in each participating borough were contacted $(n=10)$ along with all LPC leads ( $n=4$ as one covers 2 LPC areas), and 2 participating $(P)$ and 2 non-participating 
(NP) pharmacies per LPC area (one independent and one national chain) chosen purposively to cover a range of independents and national chains $(n=20)$ giving a sample population of 34 . Contact details for participants were provided by the project co-ordinator from Health Education England, after prior communication with them. Interviews were either conducted over the phone, using verbal consent, or at the working place of the participants, using written consent, and were conducted by one of the researchers. The interviews were digitally recorded and then transcribed verbatim into Microsoft Word for thematic analysis, prior to deletion. Participants were given a participant pseudonym at transcription to support anonymisation of data. The research team was independent to delivery of training and provision of services, and the interviewer had no established relationships with stakeholders prior to the study.

\section{Data analysis:}

Data collected from the pre and post-training questionnaires were stored using a VBA-Excel Tool, and were analysed descriptively. The responses were paired and statistical significance was established via paired t-test. The mean of Likert scale responses for confidence pre and post training was calculated.

Analysis was completed using an inductive thematic framework approach using five phases of familiarisation. ${ }^{18}$ NVIVO 10 software (QSR International Ply Ltd) was used to support this. Two researchers coded the data, then compared and modified the themes. Data saturation was achieved after the $12^{\text {th }}$ interview. However, all planned interviews were completed to represent all stakeholders.

This research was approved by a University Ethics Committee (1213/081). 


\section{Results}

\section{Response Rate}

The pre-training evaluation received a $100 \%(n=354)$ response rate. Of the 282 $(80 \%)$ participants eligible to take part in the post-training evaluation a response rate of $18.8 \%$ ( $n=53 / 282)$ was obtained. Responses represented national chains and independent pharmacies with $26 \%$ of responses from national chain pharmacies (14/53). Response rates varied by LPC with Bexley and Greenwich at $28 \%(n=15)$, and Kingston and Richmond at 3\% $(n=1)$.

\section{Pre-training Evaluation Results}

Participants saw the role of a $\mathrm{HC}$ as providing promotion of a healthy lifestyle by imparting health advice to the public. Others perceived it as an educational role to enable the public to make informed choices about their health or signposting as appropriate. Increasing knowledge, awareness, helping people access and use services and supporting disease prevention and positive behaviour change were all perceived as part of the role.

Most $(90 \%, n=319$ and $89 \%, n=315)$ felt that becoming a qualified $\mathrm{HC}$ was of importance to their pharmacy and to the local community respectively. Overall, $86 \%$ $(n=304)$ agreed that such training is essential for their personal development. Over three quarters $(78 \%, n=276)$ of participants agreed that on a normal working day up to 20 opportunities for delivering healthy messages to the public were available, although $62 \%(n=224)$ made less than 10 interventions a day, with only $8 \%(n=26)$ actually making over 20 interventions per day. 
The uptake of healthy living services varied between pharmacies in different boroughs, reflective of the commissioning of services. Overall, smoking cessation had the highest uptake $(90 \%, n=319)$ daily, with alcohol services being signposting to least $(62 \%, n=219)$ daily.

With regards to primary focus public health areas for the trainees, there was not a conclusive answer given, but areas included weight management, mental health, sexual health, drug misuse, diabetes management and child care, and demonstrated an awareness of local public health issues affecting their localities.

\section{Post-training Evaluation Results}

The pre-training responses of the $53 \mathrm{HC}$ who completed the post training survey was generally reflective of the overall pre-training survey results (Tables 1-3).

Nearly all of those who responded $(83 \%, n=44)$ indicated they had engaged in their $\mathrm{HC}$ role as part of their pharmacy practice. Of the 9 who had not, 8 were from independent pharmacies. A comparison of daily health interventions showed an increase of $104.25 \%$ going from 141 to 288 across South London within one month of training, with $111.45 \%$ increase in independents and $88.89 \%$ increase in national chains. However, this difference was not statistically significant $(P=0.054)$. The mean score for confidence in the new role increased from (3.6) to (4) after training with more confidence seen in $\mathrm{HC}$ from those based in national chain pharmacies. Results can be seen in table 1 . The services $\mathrm{HC}$ felt most comfortable delivering included blood pressure monitoring $(74 \%, n=38)$, smoking cessation $(74 \%, n=39)$ and weight management $(66 \%, n=33)$. 
When comparing frequency of interventions for different services pre and post training, on a daily basis, increases were seen in all services (Table 2). However, the increase was only statistically signification in relation to the blood pressure monitoring service.

An increase was also seen after training of arrangements to visit organisations that could be used for signposting (Table 3).

The HCs perceived the benefits of the role to include self-confidence, enhanced quality of health services provision, awareness of health issues and local services, and a reduced workload for pharmacists. Challenges identified were mainly related to resources in terms of time, workload and staff, and material such as leaflets and posters, lack of commissioned services at pharmacy and lack of awareness of local services to enable signposting, inappropriate facilities, and reluctance from management to permit interactions with patients. The responders proposed continued training and periodic workshops for each borough for development, further engagement and practice with other $\mathrm{HC}$, to support the role long term.

\section{Interview Results}

22 interviews in total were undertaken, taking a maximum of 15 minutes each, with the following participation rates from each of the four categories identified: PHE $(40 \%, n=4)$, LPCs $(100 \%, n=4)$, participating pharmacies $(P)(70 \%, n=7)$, nonparticipating pharmacies (NP) $(70 \%, n=7)$ giving a total response rate of $64.7 \%$ $(n=22 / 34)$. Due to limited sample populations, demographic data was not captured in order to maintain anonymity of responders.

The 2 key themes identified from the interviews were: Training and Impact. 
The need for training of support staff was clearly identified (Box 1.1-1.4). The HC training was reported as well received with overall satisfaction with the quality and quantity of training delivery (Box 1.5-1.7). Some felt that training should follow commissioning and not be blanket training (Box 1.8-1.9) as commissioned services varied by borough, as echoed in the survey data.

Therefore, more sessions were needed to provide more information about local services (Box 1.10). Barriers for participation in training included lack of communication (Box 1.11) as well as staff and time constraints (Box 1.12-1.14). Suitable times and local training were seen to enable uptake (Box 1.15). Follow up is also required (Box 1.16) in part due to potential staff turnover or migration (Box 1.17). Support from pharmacists is seen as supporting success of the role (Box 1.181.19).

When talking about impact, $\mathrm{HC}$ were deemed as valuable to the community providing public health advice by the interviewed stakeholders (Box 2.1-2.2) as well as having an impact on local commissioning of services (Box 2.3). A large impact after the training was also the sense of job satisfaction, with the ability to deliver services through increased confidence and knowledge, and an expanded role (Box 2.4-2.9).

\section{Discussion}

This study shows stakeholder perspectives on $\mathrm{HC}$ training and role and gives the largest sample of $\mathrm{HC}$ perspectives to date. It showed that $\mathrm{HCs}$ post training were actively engaged in the role with the number of blood pressure monitoring 
interventions statistically increased. The interviewed pharmacists felt that the training enhanced HCs job satisfaction and ability to deliver public health services.

Limitations of the study are that it was carried out in only one area so may not reflect practice or services in other areas, with a very poor response rate for the post evaluation survey limiting statistical comparisons.. Therefore, although responses from pre-training survey can be generalised the post-training results cannot. Furthermore, the small number of responders may constitute mostly of those who were proactive in their role.

Positivity and increased confidence towards becoming a $\mathrm{HC}$ echoes a previous qualitative study with $\mathrm{HCs}$ post training. ${ }^{19}$ However, it is evident that more time is needed to assess the impact on pharmacy engagement with public health and the resulting changes for the members of the public in the community who use these services. The importance of matching services to the needs of each locality was highlighted as well as the conjunction of further training for $\mathrm{HC}$, targeted at tackling their area's local public health issues.

From this study, it is seen that community pharmacy currently provides a range of public health services, which vary according to location, and there is potential to support further interventions, perhaps through greater analysis of annual patient questionnaires. This is supported by a review of the literature about HLPs previously conducted..$^{20}$ However, as per a previous study, for success, more work needs to be done to integrate factors such as pharmacy environment, support staff and 
collaboration with external stakeholders to maximise utilisation and impact, through signposting. ${ }^{21}$

Pharmacists and $\mathrm{HC}$ themselves saw the role as a positive asset. Patients potentially also benefit from the role with high levels of activity in new roles seen, with increased awareness of signposting and improved confidence. This has also been seen in a previous qualitative study of HCs in HLPs. ${ }^{22}$ It is important, that, as UK healthcare policy and planning continues to value the role of pharmacy in delivering public health, that all of the pharmacy team continue to work together to provide and contribute to new public health initiatives, recognising the important role of support staff, alongside pharmacists. ${ }^{23}$ The latter has been echoed by the interview results.

Engagement barriers stemmed from busy schedules of pharmacies with low staff availability for covering those who were able to attend training. Logistically this is unsurprising given how busy community pharmacies can be. It should be noted that by offering additional training opportunities over time, more pharmacies will have the opportunity to participate.

The training increased HCs job satisfaction. Studies by Deeks et.al. ${ }^{23}$ and Brown et.al. ${ }^{15}$ both confirm the findings that giving more training and responsibility to staff in public health roles increases their job satisfaction. In both elements of the study it was seen that training should be localised to match local needs and commissioned services. Therefore, the continual local support of the programme is important to provide on-going training to avoid loss of satisfaction and decreased engagement as echoed by Nazar et al. ${ }^{24}$ As part of the training, all stakeholders were briefed about the programme prior to commencement. Involving pharmacists and pharmacy 
managers in training can help them help them understand the role and would be of benefit. $^{25}$

Following the Portsmouth pilot success, non-HLPs in the area were motivated to gain HLP status. ${ }^{15}$ Although many champions were trained in South London, more time will be needed to assess the impact of local HLP accreditation because of having a $\mathrm{HC}$ in place.

Future training should ensure local factors are taken into account, and the initial training for $\mathrm{HC}$ is followed by specific training on the topics they see in their pharmacies with buy in from local stakeholders.

\section{Conclusion}

Preliminary results demonstrated an overall positive impact of training on confidence of $\mathrm{HC}$ in healthcare provision, improved awareness in local signposting and an overall increase in the number of daily health interventions made by staff. The lack of statistical significance indicates the need for repeating this part of the study, but should not deduct from the positive feedback indicated by those who did participate. The programme is only successful where pharmacists give their full support. Training should have a structured evaluation strategy of the change in role following training. The overall study demonstrates the utility of community pharmacy in public health provision with initiatives such as HLP driving increases in patient interventions. By ensuring programmes such as HLP use support from policy to coordinate the ongoing relationship between pharmacy and public health, this area will continue to improve. 
References:

1. Anderson S. Community pharmacy and public health in Great Britain, 1936 to 2006: how a phoenix rose from the ashes. J Epidemiol Comm health $2007 ; 61: 844-848$

2. Department of Health. Our Healthier Nation; 1998. [Online].

https://www.gov.uk/government/uploads/system/uploads/attachment data/file/ 265721/title.pdf (accessed 12.01.2017)

3. Department of Health. A Vision for Pharmacy in the New NHS. [Online] 2003. http://webarchive.nationalarchives.gov.uk/+/www.dh.gov.uk/en/Consultations/ Closedconsultations/DH_4068353 (Accessed 03 August 2014)

4. Department of health. Choosing Health Through Pharmacy: A Programme for Pharmaceutical public health 2005-2015. London, UK: Department of Health; 2005

5. NHS Choices. History of the NHS. [Online] 2014 http://www.nhs.uk/Tools/Documents/HistoryNHS.html (Accessed 20 August 2014)

6. Local Government Association. Community pharmacy. Local government's new public health role. [Online] 2013

http://www.local.gov.uk/documents/10180/11463/Community+Pharmacy++local+government's+new+public+health+role/01ca29bf-520d-483e-a703$\underline{45 a c 4 f e 0 f 521}$ (accessed 03 August 2014)

7. NHS England, Public Health England, Health Education England, Monitor, Care Quality Commission, NHS Trust Development Authority Five Year Forward View. [Online] 2014 http://www.england.nhs.uk/wpcontent/uploads/2014/10/5yfv-web.pdf (accessed 30 Oct 2014) 
8. Brown TJ, Todd A, O’Malley C, et al. Community pharmacy-delivered interventions for public health priorities: a systematic review of interventions for alcohol reduction, smoking cessation and weight management, including meta-analysis for smoking cessation. BMJ Open 2016;6:e009828. doi:10.1136/bmjopen-2015-009828

9. Strand $\mathrm{M}$ et al. The achievement of public health services in pharmacy practice: A literature review. Res Social Adm Pharm 2016; 12: 28247-256

10. Faculty of Public Health of the Royal Colleges of Physicians of the United Kingdom. Food Poverty and Health. [Online] 2005. May http://www.fph.org.uk/uploads/bs_food_poverty.pdf (Accessed 20 August 2014)

11. Department of Health. Building on the Best - Choice, Responsiveness and Equity in the NHS. [Online] 2003.

\section{https://www.gov.uk/government/uploads/system/uploads/attachment data/file/} 272092/6079.pdf (accessed 03 August 2014)

12. Department of Health. Pharmacy in England: Building on strength, Delivering the future. [online] 2008

https://www.gov.uk/government/uploads/system/uploads/attachment data/file/ 228858/7341.pdf. (accessed 18 August 2014)

13. National Pharmaceutical Association. HLP overview [online]. http://www.npa.co.uk/Documents/HLP/HLP overview 12.11.pdf. $\quad$ (accessed 27 August 2014)

14.Duggan, C., Evans, D (2013) Evaluation of the Healthy Living Pharmacy Pathfinder Work Programme 2011-2012, UCL Institute of Health Equity. 
15. Brown D et.al. From community pharmacy to healthy living pharmacy: Positive early experiences from Portsmouth, England. Res Social Adm Pharm 2014; 10:72-87

16.Donovan G, Paudyal V. England's Healthy Living Pharmacy (HLP) initiative: Facilitating the engagement of pharmacy support staff in public health. Res Social Adm Pharm 2016; 12: 281-292

17. Patel $\mathrm{R}$ et al. The experience of healthy living pharmacies within Lambeth. Int J Pharm Pract 2014; 22:47

18.Braun V, Clarke V. Using thematic analysis in psychology. Qual Res Psychol 2006: 3(2):77-101.

19. Rutter P, Vryaparj G. Qualitative exploration of the views of healthy living champions from pharmacies in England. Inj J Clin Pharm 2015; 37:27-30

20. Brown D et al. Review of services provided by pharmacies that promote healthy living. Inj J Clin Pharm 2012; 34:399-409

21. Saramunee $\mathrm{K}$ et al. How to enhance public health service utilization in community pharmacy? General public and health providers' perspectives. Res Social Adm Pharm 2014;10:272-284

22. Cooper R, Tsoneva, J. Benefits and tensions in delivering public health in community pharmacies - a qualitative study of health living pharmacy staff champions. Int J Pharm Pract (accessed 23 December 2016, epub ahead of print).

23. Deeks $L$ et al. Can pharmacy assistants play a greater role in public health programs in community pharmacies? Lessons from a Chlamydia screening study in Canberra, Australia. Res Social Adm Pharm 2013;1-6 
24. Nazar Z et al. Healthy living champions network: An opportunity for community pharmacy's sustained participation in tackling local health inequalities. Res Social Adm Pharm 2016; 12: 1010-1015

25. Eades $C$ et al. Public health in community pharmacy: A systematic review of pharmacist and consumer views. BMC Public Health 2011; 11: 582

26. The guardian. Indices of multiple deprivation: find the poorest places in England. [Online] 2011

http://www.theguardian.com/news/datablog/2011/mar/29/indicesmultipledeprivation-poverty-england (accessed 27 August 2014) 